\title{
Magnetic domain structure in thin CoPt perpendicular magnetic anisotropy films
}

\author{
Y. Kawada ${ }^{1}$, M. Onose ${ }^{1}$, R. Tojo ${ }^{1}$, T. Komine ${ }^{1}$, and R. Sugita ${ }^{1}$ \\ ${ }^{1}$ Media and Telecommunications Engineering, Ibaraki University, 4-12-1 Nakanarusawa-cho, Hitachi, Ibaraki 316- \\ 8511 , Japan
}

\begin{abstract}
The relation between thickness and domain structure of $\mathrm{Co}_{80} \mathrm{Pt}_{20}$ perpendicular magnetic anisotropy films was investigated through experiments and micromagnetic simulation. The films with thickness over $10 \mathrm{~nm}$ exhibited clear maze domain structure, while for the films thinner than $10 \mathrm{~nm}$ the domain structure abruptly changed from maze domain to irregular and large domain as the thickness became thinner. The irregular domain had narrower domain wall width than maze domain.
\end{abstract}

\section{Introduction}

CoPt-based films with high perpendicular magnetic anisotropy are suited to the recording layer of hard disks [1-3]. It is important to elucidate magnetic domain structure of the films because it directly relates to SN ratio [4-6]. And it was reported that the domain structure of $L 1_{1}$ CoPt films changed from maze domain to irregular and larger one as the film thickness became thinner [7]. However, the relation between thickness and domain structure for $\mathrm{Co}_{80} \mathrm{Pt}_{20}$ films has not been discussed yet in detail, and relation between domain structure and magnetization state has not been mentioned yet. In this study, we investigated the influence of thickness on domain structure of $\mathrm{Co}_{80} \mathrm{Pt}_{20}$ films especially in thin film thickness region with experiment, and analyzed magnetization state with micromagnetic simulation.

\section{Experimental simulation method}

$\mathrm{Co}_{80} \mathrm{Pt}_{20} / \mathrm{Ru}$ (thickness of $\left.20 \mathrm{~nm}\right) / \mathrm{Pt}(100 \mathrm{~nm})$ films were deposited on glass substrate using an RF-magnetron sputtering system. Thickness was varied from 3 to $50 \mathrm{~nm}$. No substrate heating was carried out during each deposition process. The Ar pressure during film deposition was $2 \times 10^{-3}$ Torr for all films. Magnetic properties were measured with a vibrating sample magnetometer (VSM). Domain structure was evaluated using a magnetic force microscope (MFM).

In order to investigate the influence of CoPt film thickness on domain structure and size, micromagnetic simulation was carried out. The equilibrium magnetization distribution of CoPt films was solved by Landau-Lifshitz-Gilbert (LLG) equation, which was
Table 1. Magnetic parameters of CoPt film.

\begin{tabular}{|c|c|c|}
\hline $\begin{array}{c}\text { Saturation } \\
\text { magnetization } \\
M_{\mathrm{S}}\left(\mathrm{emu} / \mathrm{cm}^{3}\right)\end{array}$ & $\begin{array}{c}\text { c-axis } \\
\text { distribution } \\
\Delta \theta_{50}(\mathrm{deg} .)\end{array}$ & $\begin{array}{c}\text { Exchange constant } A \\
\left(\times 10^{-6} \mathrm{erg} / \mathrm{cm}\right)\end{array}$ \\
\hline 1300 & 5 & $\begin{array}{c}1 \text { (for } 5 \mathrm{~nm} \text { thick film) } \\
3 \text { (for } 10,20,50 \mathrm{~nm} \text { thick films) }\end{array}$ \\
\hline
\end{tabular}

Table 2. Averaged anisotropy field $H_{\mathrm{k}}$ for CoPt films with various thickness.

\begin{tabular}{|c|c|c|c|c|}
\hline $\begin{array}{c}\text { Thickness } \\
(\mathrm{nm})\end{array}$ & 5 & 10 & 20 & 50 \\
\hline$H_{\mathrm{k}}(\mathrm{kOe})$ & 17 & 16 & 15 & 11 \\
\hline
\end{tabular}

originally coded by us [8]. The calculation region is 600 $\mathrm{nm} \times 600 \mathrm{~nm} \times \delta_{\mathrm{CoPt}} \mathrm{nm}$ and was divided into $5 \mathrm{~nm} \times 5$ $\mathrm{nm} \times 5 \mathrm{~nm}$ cubic cells, where $\delta_{\mathrm{CoPt}}$ is the film thickness and was varied from 5 to $50 \mathrm{~nm}$. The magnetic parameters which were used for the simulation are shown in Table 1. It was reported that the magnetic anisotropy $K_{\mathrm{u}}$ increased as the film thickness decreased, and $K_{\mathrm{u}}$ was related to a lattice deformation [9-11]. From the reports, anisotropy field $H_{\mathrm{k}}$ was set to increase as the thickness decreased in the simulation. Table 2 shows the averaged $H_{\mathrm{k}}$ for CoPt films with various thickness.

\section{Results and discussion}

\subsection{Experimental results}

Fig. 1 shows perpendicular hysteresis loops of CoPt films with various thickness. Saturation magnetic field $H_{\mathrm{s}}$ and nucleation field $H_{\mathrm{n}}$ obtained from the perpendicular hysteresis loops are summarized in Fig. 2 as a function of 


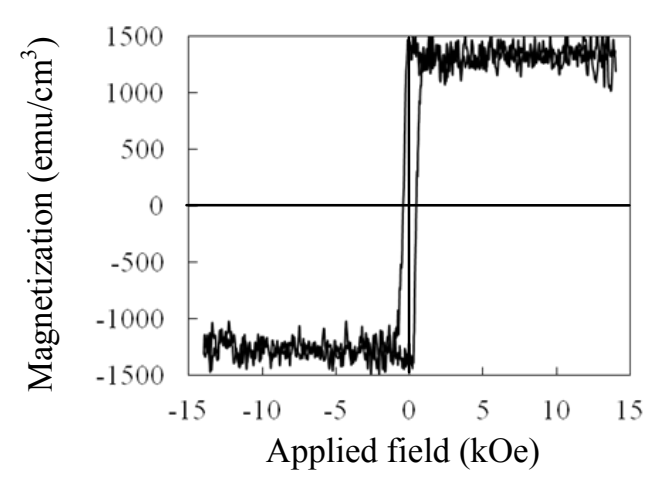

(a) Thickness $=5 \mathrm{~nm}$

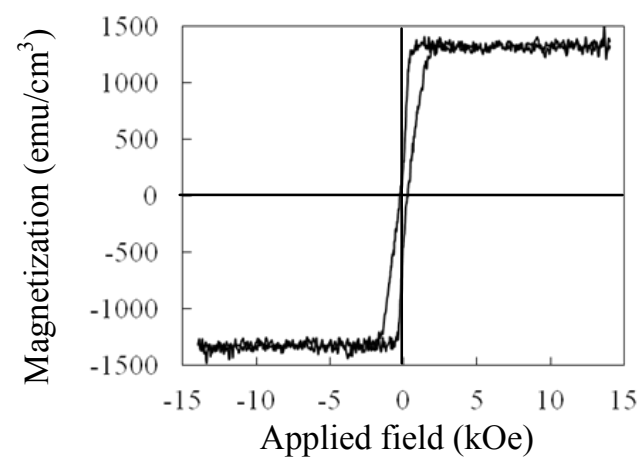

(b) $10 \mathrm{~nm}$

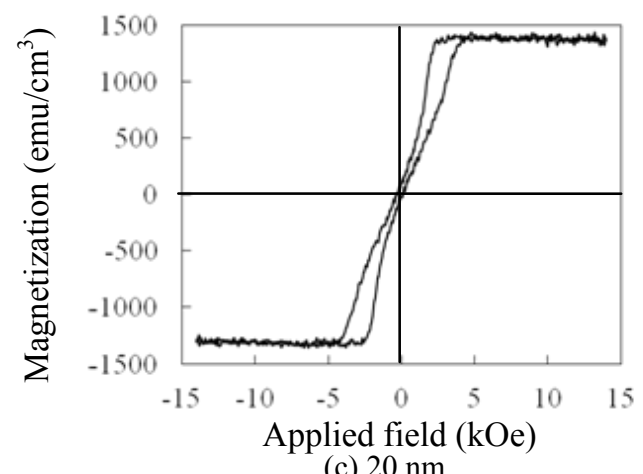

Fig. 1. Perpendicular hysteresis loops of CoPt films with different thickness.

film thickness. From Figs. 1 and 2, it is found that the $H_{\mathrm{s}}$ decreases as the thickness decreases and that for thicker films, $H_{\mathrm{n}}$ is positive and reduces with decrease in thickness down to a thin film thickness of about $10 \mathrm{~nm}$ and for thinner films $H_{\mathrm{n}}$ becomes more and more negative. Origins of the decrease of $H_{\mathrm{s}}$ and $H_{\mathrm{n}}$ with decrease of film thickness are considered to be due to the increase of perpendicular magnetic anisotropy and an increasing impedance of domain wall motion with decrease in film thickness [7, 9-12].

Fig. 3 shows MFM images of the CoPt films shown in Fig. 1. Samples for the MFM observation were prepared as follows. After perpendicular field of $15 \mathrm{kOe}$ was applied to the films, the field was changed to the remanent coercivity, then the field was removed. The MFM images after DC-demagnetization, ACdemagnetization and virgin state exhibited similar domain structure. The films with thickness of 10 and 20 $\mathrm{nm}$ exhibit maze domain structure, while the $5 \mathrm{~nm}$ thick film shows large and irregular domain [7]. The

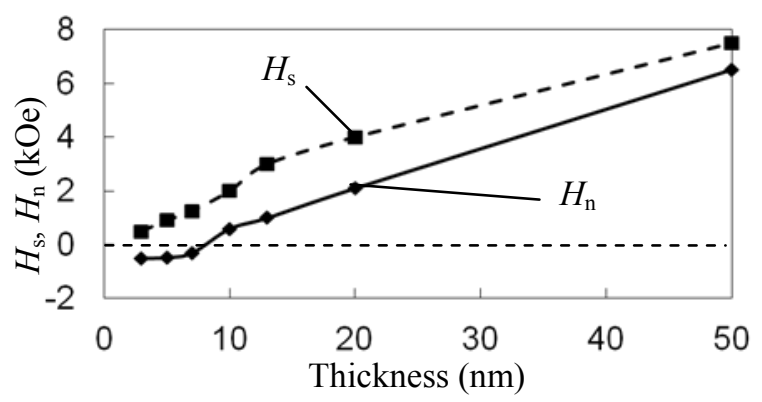

Fig. 2. Dependence of $H_{\mathrm{s}}$ and $H_{\mathrm{n}}$ on thickness of CoPt films.
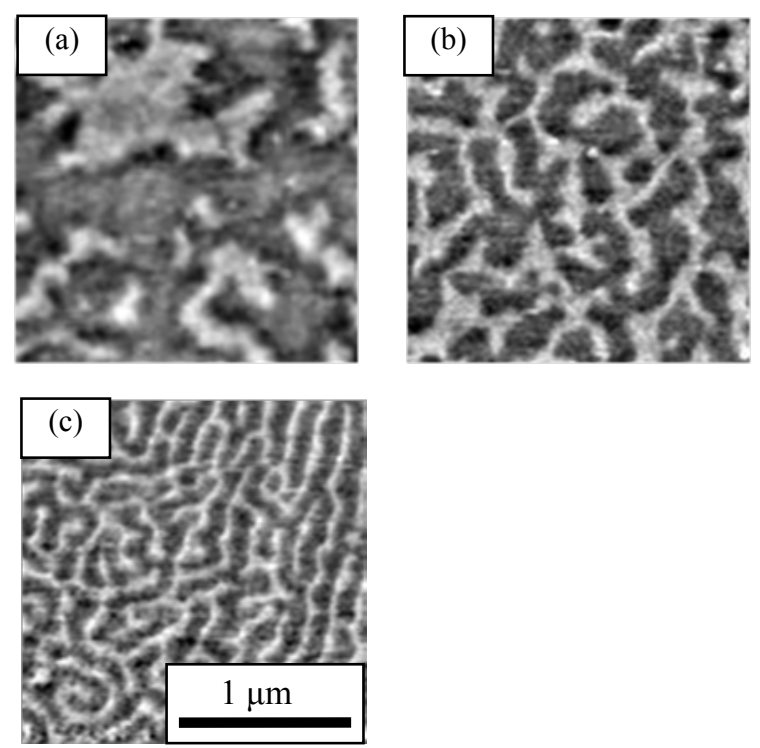

Fig. 3. MFM images of CoPt films with thickness of (a) $5 \mathrm{~nm}$, (b) $10 \mathrm{~nm}$, and (c) $20 \mathrm{~nm}$.

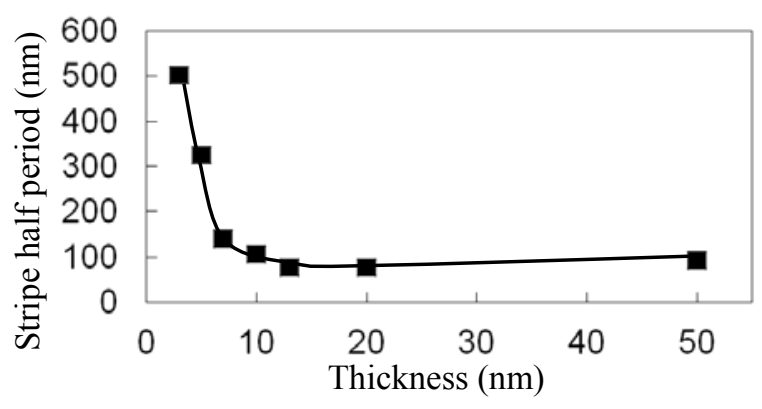

Fig. 4. Stripe half period as a function of film thickness.

squareness ratio of the perpendicular hysteresis loops of the films having irregular domain structure such as Fig. 3 (a) was 1.0. Fig. 4 shows averaged stripe half period (SHP) estimated from MFM images as a function of film thickness. For the films less than $13 \mathrm{~nm}$ the SHP becomes smaller as film thickness becomes thicker, while for the films more than $13 \mathrm{~nm}$ the SHP becomes slightly larger as the thickness becomes thicker. It has been shown that the increasing SHP with increase of thickness can be expressed as follows [13].

$\mathrm{SHP} \propto \sqrt{t \times D_{0}}$ for $t \geq D_{0}$

where $t$ is film thickness, $D_{0}$ is dipolar length which is a balance between domain wall energy and demagnetizing energy, and is defined as 


$$
D_{0}=\sigma_{w} / 2 \pi M_{s}^{2}
$$

where $\sigma_{\mathrm{w}}$ is wall energy density. The tendency of the SHP more than $13 \mathrm{~nm}$ shown in Fig. 4 is consistent with Eq. (1). On the other hand, Kaplan and Gehring [14] derived an expression for films with thickness below $D_{0}$ as

$$
\mathrm{SHP} \propto t \times e^{\pi D_{0} / 2 t} \text { for } t \leq D_{0} .
$$

From Eq. (3), it is found that the SHP becomes smaller as the thickness becomes thicker in thin thickness region. The tendency of the SHP less than $13 \mathrm{~nm}$ is in agreement with Eq. (3). It is inferred that $D_{0}$ for the films shown in Fig. 4 is around $13 \mathrm{~nm}$.

\subsection{Simulation and discussion}

Fig. 5 shows simulated perpendicular hysteresis loops of CoPt films with various thickness. Each shape of hysteresis loops almost coincides with the experimental

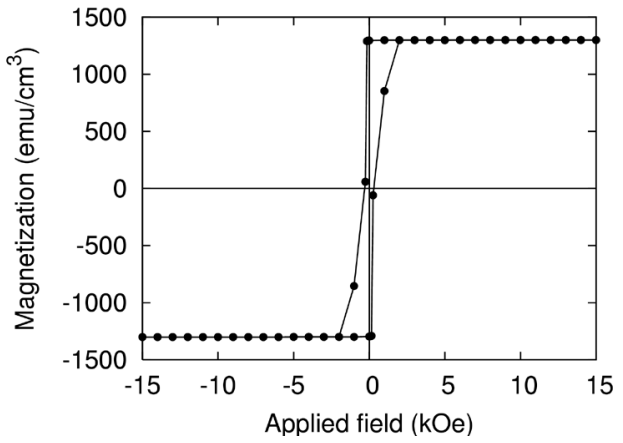

(a) Thickness $=5 \mathrm{~nm}$

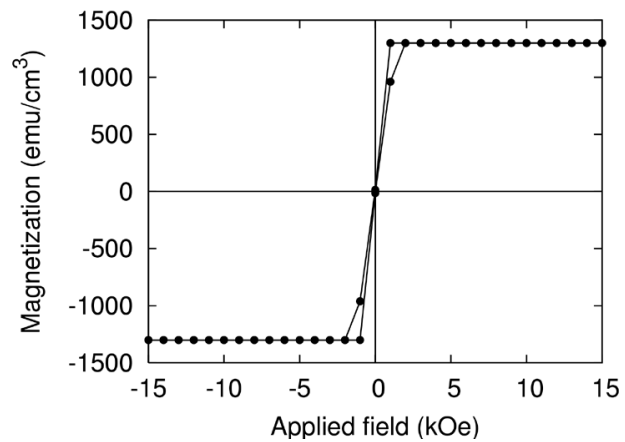

(b) $10 \mathrm{~nm}$

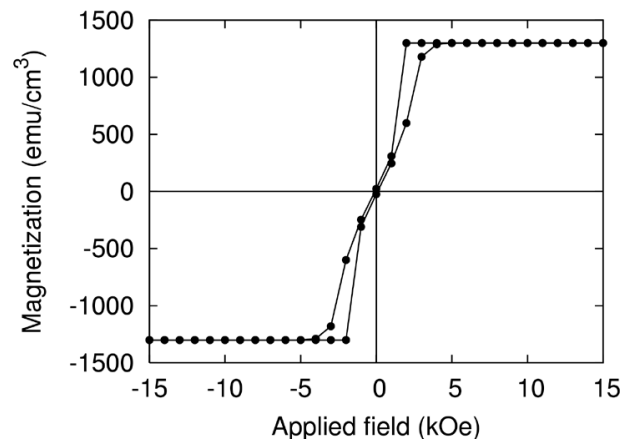

(c) $20 \mathrm{~nm}$

Fig. 5. Simulated perpendicular hysteresis loops of CoPt films with different thickness. one shown in Fig. 1. $H_{\mathrm{s}}$ and $H_{\mathrm{n}}$ increase as the thickness increases. Because the simulated hysteresis loops almost coincide with the experimental ones, it seems to be appropriate to make consideration on domain structure obtained from the experiment by using the simulation with the parameters shown in Tables 1,2 .

Fig. 6 shows simulated magnetization distribution of CoPt films with various thickness after DCdemagnetization. White areas represent $M_{\mathrm{z}} / M_{\mathrm{s}}=1$ and black areas represent $M_{\mathrm{z}} / M_{\mathrm{s}}=-1$. Magnetization distribution of the $20 \mathrm{~nm}$ thick CoPt film exhibits maze domain structure, while the $5 \mathrm{~nm}$ thick film shows large and irregular-like domain. Fig. 7 shows averaged SHP estimated from simulated magnetization distribution as a function of film thickness. For the films less than $20 \mathrm{~nm}$ the SHP becomes smaller as film thickness becomes thicker, while the $50 \mathrm{~nm}$ thick film has larger SHP than the $20 \mathrm{~nm}$ thick film. The domain structure and the tendency of the SHP are almost in agreement with the experimental results shown in Figs. 3 and 4. Fig. 8 shows cross section of the simulated magnetization distribution near domain wall. In Fig. 8, the length of the magnetization vector is expressed as $\left(M_{\mathrm{z}}^{2}+M_{\mathrm{x}}^{2}\right)^{1 / 2}$, so short magnetization vector indicates that the magnetization inclines to $y$-direction. From Fig. 8, it is found that the film with thickness of $5 \mathrm{~nm}$ which exhibits irregular-like domain has narrower domain wall width than the films exhibiting maze domain.

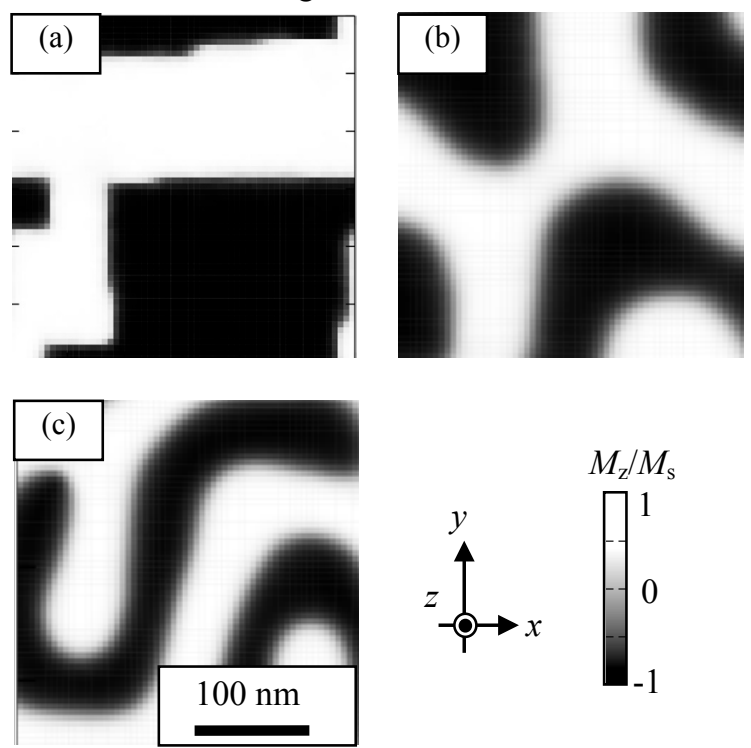

Fig. 6. Simulated magnetization distribution of CoPt films with thickness of (a) $5 \mathrm{~nm}$, (b) $10 \mathrm{~nm}$, and (c) $20 \mathrm{~nm}$.

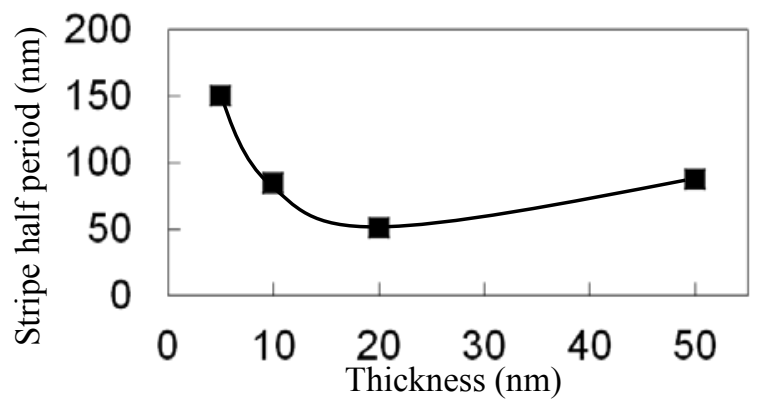

Fig. 7. Simulated stripe half period as a function of film thickness. 


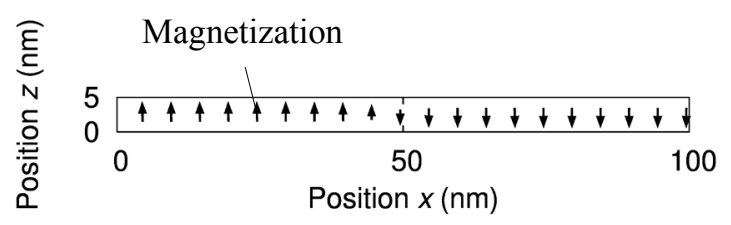

(a) Thickness $=5 \mathrm{~nm}$

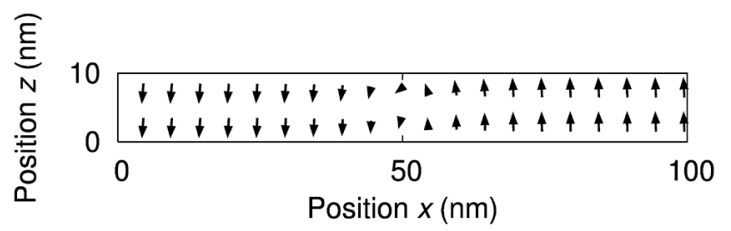

(b) $10 \mathrm{~nm}$

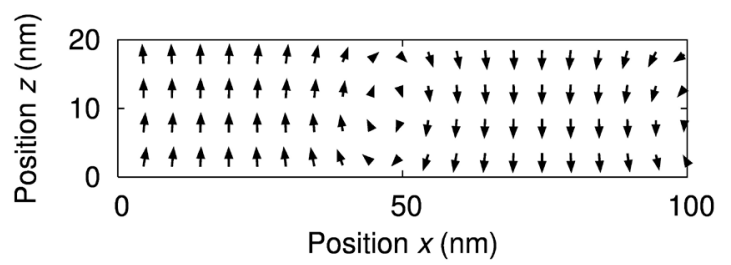

(c) $20 \mathrm{~nm}$

Fig. 8. Cross-sectional view of simulated magnetization distribution of CoPt films with thickness of (a) 5, (b) 10, and (c) $20 \mathrm{~nm}$.

\section{Conclusion}

In this study, we investigated the influence of thickness on domain structure of $\mathrm{Co}_{80} \mathrm{Pt}_{20}$ films with experiment, and analyzed magnetization state with micromagnetic simulation. As a result, for the films with thickness thinner than $10 \mathrm{~nm}$, the domain structure abruptly changed from maze domain to irregular domain. The simulation showed irregular-like domain has narrower domain wall width than maze domain.

\section{Acknowledgment}

This work was supported in part by the Grant-in-Aid for Scientific Research (C) (No. 24560394) from the Japan Society for the Promotion of Science (JSPS) of Japan.

\section{References}

1. Y. Wang, J. Ariake, T. Wang, S. Watanabe, N. Honda, F. S. Li, K. Ouchi, J. Appl. Phys., 107, 103925 (2010).

2. S. H. Park, T. D. Lee, S. H. Kong, S. Y. Yoon, H. S. Lee, H. J. Kim, H. S. Oh, J. Appl. Phys. 103, 07F528, (2008).

3. D. E. Laughlin, Y. Peng, Y.-L. Qin, M. Lin, and J.-G. Zhu, IEEE Trans. Magn., 43, 693 (2007).

4. M. Hashimoto, K. Miura, H. Muraoka, H. Aoi, and Y. Nakamura, IEEE Trans. Magn., 39, 2624 (2003).

5. M. Hashimoto, K. Miura, H. Muraoka, H. Aoi, and Y. Nakamura, IEEE Trans. Magn., 40, 2458 (2004).

6. M. Hashimoto, K. Miura, H. Muraoka, H. Aoi, and Y. Nakamura, J. Magn. Magn. Mater., 287, 123 (2005).

7. Y. Yang, J. S. Chen, G. M. Chow, J. Appl. Phys., 109, 07B744 (2011).

8. T. Komine, H. Murakami, T. Nagayama, and R. Sugita, IEEE Trans. Magn., 44, 2516 (2008).

9. T. Shimatsu, H. Sato, Y. Okazaki, H. Aoi, H. Muraoka, Y. Nakamura, S. Okamoto, O. kitakami, J. Appl. Phys., 99, 08G908 (2006).

10. T. Shimatsu, Y. Okazaki, H. Sato, O. kitakami, S. Okamoto, H. Aoi, H. Muraoka, and Y. Nakamura, IEEE Trans. Magn., 43, 2995 (2007).

11. T. Shimatsu, Y. Okazaki, H. Sato, H. Aoi, J. Appl. Phys., 103, 07F524 (2008).

12. J.-U. Thiele, L. Folks, M. F. Toney, and D. K. Weller, J. Appl. Phys., 84, 5686 (1998).

13. C. Kooy and U. Enz, Philips Res. Rep 15, 7 (1960).

14. B. Kaplan, G. A. Gehring, J. Magn. Magn. Mater., 128, 111 (1993). 